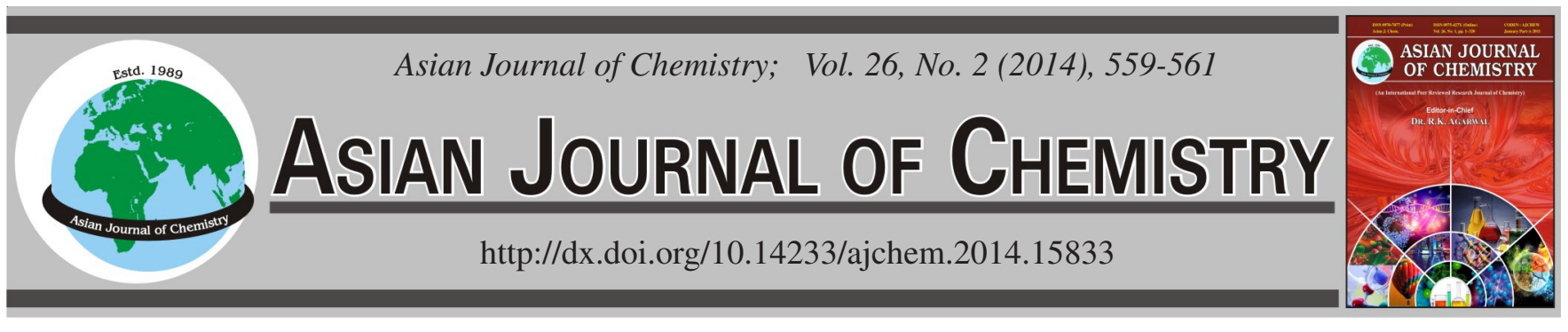

\title{
Determination of Antibacterial Activity of Aucubigenin and Aucubin
}

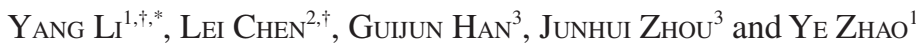

${ }^{1}$ College of Life Sciences, Northwest University; Biomedicine Key Laboratory of Shaanxi Province, Xi'an 710069, Shaanxi Province, P.R. China ${ }^{2}$ Department of Pharmacy, Xijing Hospital, Fourth Military Medical University, Xi'an, 710032, Shaanxi Province, P.R. China

${ }^{3}$ Xi'an Botanical Garden, Institute of Botany of Shaanxi Province, Xi'an 710061, Shaanxi Province, P.R. China

*Corresponding author: E-mail: ly2011@nwu.edu.cn

$\dagger$ These authors contributed equally to this work.

\begin{abstract}
Because of the previously conflicted reports, the aucubigenin and aucubin have been reinvestigated for their antibacterial activities by a sensitive standardized micro-well dilution with automated spectrophotometric method, compared with paper disc diffusion method. The micro-well dilution assay was more accurate than paper disc diffusion method. The results showed that aucubin exhibited no antibacterial activity, while aucubigenin showed remarkable antibacterial activity against all of the tested bacteria. Aucubigenin was particularly more effective against Staphylococcus aureus with MIC value of $0.0313 \mathrm{mg} / \mathrm{mL}$ and MBC value of $0.0625 \mathrm{mg} / \mathrm{mL}$, respectively.
\end{abstract}

Keywords: Aucubigenin, Aucubin, Antibacterial Activity, Minimum inhibitory concentration, Minimum bacterial concentration. ᄂ - - - - - - - - - - - - - - - - - - - - - - - - - - - -

\section{INTRODUCTION}

The search for natural antibacterial compounds, especially of plant origin, has greatly increased in recent years. Numerous crude extracts and pure compounds from natural plants were reported to have antibacterial activity ${ }^{1,2}$. Our Study on the chemical constituents of total glycosides from the seeds of Eucommia ulmoides Oliv caused the isolation of the main iridoid glycoside aucubin ${ }^{3}$. Aucubigenin, which is the aglycone of aucubin, is an iridoid compound characterized by high instability ${ }^{4}$.

Previous studies have reported the antibacterial properties of aucubigenin and aucubin showing minimum inhibitory concentration (MIC) values ${ }^{5,6}$. However, in a discussion on antibacterial properties of aucubigenin, Davini et $a l^{6}$ reported that it was effective against Escherichia coli, Staphylococcus aureus and Pseudomonas aeruginosa with MIC values of 8, 1 and $4 \mathrm{mg} / \mathrm{mL}$, respectively. But Ishiguro et al. ${ }^{5}$ reported the MIC values of the above three tested strains were $>100,6.25$ and $>100 \mu \mathrm{g} / \mathrm{mL}$, respectively. These two reports contrasted starkly with remarkable difference in MIC, which appeared to be in conflict and reflects continuing uncertainty. Furthermore, Ishiguro et $a .^{5}{ }^{5}$ used aucubigenin produced by incubation of aucubin with $\beta$-glucosidase and without isolation. This was rather inaccurate since the solution containing a mixture of aucubigenin and aucubin was directly applied to antibacterial test.
Herein the antibacterial activities of aucubigenin and aucubin were obtained using a sensitive standardized method, 96-well microplate measuring absorbance with automated microplate spectrophotometer. This spectrophotometric method of reading MICs is rapid, reproducible, free from subjectivity and observer variability and more accurate than paper disc diffusion method ${ }^{7,8}$. In addition, we used pure product of aucubigenin and aucubin, which allowed us to determine accurately the MIC and minimum bactericidal concentration (MBC) values.

\section{EXPERIMENTAL}

Aucubigenin, colorless and transparent crystals, obtained from the enzymatic hydrolysis of aucubin by $\beta$-glucosidase. Colourless and transparent crystals of aucubin were isolated from the seeds of Eucommia ulmoides Oliv in our laboratory (Biomedicine Key Laboratory of Shaanxi Province, Northwest University).

Microbial strains: Aucubigenin and aucubin were individually tested against a panel of four microorganisms. Following bacterial strains were provided by Molecular Microbiology Laboratory, College of Life Sciences, Northwest University, Xi' an, China: Escherichia coli (ATCC 25922), Staphylococcus aureus (ATCC 25923), Pseudomonas aeruginosa (ATCC 27853) and Salmonella enterica (ATCC 14028). Bacterial strains were cultured overnight at $37{ }^{\circ} \mathrm{C}$ in on Luria-Bertani (LB) agar plates. 
Inhibitory effect by the disc diffusion method: The paper disc diffusion method was employed for the determination of the antibacterial activity of aucubigenin and aucubin ${ }^{9}$. The samples were dissolved in $50 \% \mathrm{MeOH}$ to a final concentration of $10 \mathrm{mg} / \mathrm{mL} .100 \mu \mathrm{L}$ of suspension containing $10^{8} \mathrm{CFU} / \mathrm{mL}$ of bacteria spread on the $20 \mathrm{~mL}$ of LB mediums. Filter paper discs (6 mm in diameter) impregnated with $10 \mu \mathrm{L}$ of sample solution and the same volume of $50 \% \mathrm{MeOH}$ was used as a negative control. After incubating the plates for $24 \mathrm{~h}$ at $37^{\circ} \mathrm{C}$, antibacterial activity was evaluated by measuring the inhibition zone.

Inhibitory effect by micro-well dilution assay: Aucubigenin and aucubin were studied for their MIC and MBC values using micro-well dilution assay method ${ }^{10}$. The samples dissolved in $\mathrm{MeOH}$ solution were first diluted to the highest concentration $(8 \mathrm{mg} / \mathrm{mL})$ to be tested and then serial two fold dilutions were made in a concentration range from 0.0313 to $8 \mathrm{mg} / \mathrm{mL}$ of the two samples in a 96-well microtitre plate, including one growth control (LB + bacteria) and one sterility control (LB + test sample). $90 \mu \mathrm{L}$ of LB was added onto the microplates with $5 \mu \mathrm{L}$ of the tested solution. Then, $5 \mu \mathrm{L}$ of standard microorganism suspensions were inoculated onto the microplates. Tetracycline was used as standard drugs for positive control in conditions identical to tests materials. The contents of each well were mixed on a plate shaker at $300 \mathrm{rpm}$ for $20 \mathrm{~s}$ and then incubated at the appropriate temperature for $24 \mathrm{~h}$. Microbial growth was determined by absorbance at 600 $\mathrm{nm}$ using the Wallac 1420 Victor multilabel counter (PerkinElmer). The MIC of each compound was defined as the lowest concentration that inhibited microorganism growth. The MBC was determined by sub-culturing $10 \mu \mathrm{L}$ of the MIC test solutions on nutrient agar plate at $37{ }^{\circ} \mathrm{C}$ for $24 \mathrm{~h}$. The highest dilution that yielded no bacterial growth was taken as $\mathrm{MBC}^{11}$.

Statistical analysis: All the experiments were done in triplicate. The data were recorded as mean \pm standard deviations (SD). Analysis of variance was performed by One-way ANOVA procedure of PASW Statistics 18 (SPSS version 18.0 for Windows, SPSS Inc., Chicago, USA).

\section{RESULTS AND DISCUSSION}

In our paper disc diffusion method experiment, we noted that the inhibition zones of aucubigenin were visible at the end of the incubation period after $24 \mathrm{~h}$, but they were very difficult to measure. Because around the paper discs a soluble dark brown colour had diffused into the medium to make the edge blurred. Due to this, the diameters of the inhibition zones could not be measured or were not measured correctly and accurately (Fig. 1).

The MIC and MBC of aucubigenin and aucubin using micro-well dilution assay method are shown in Table-1. The

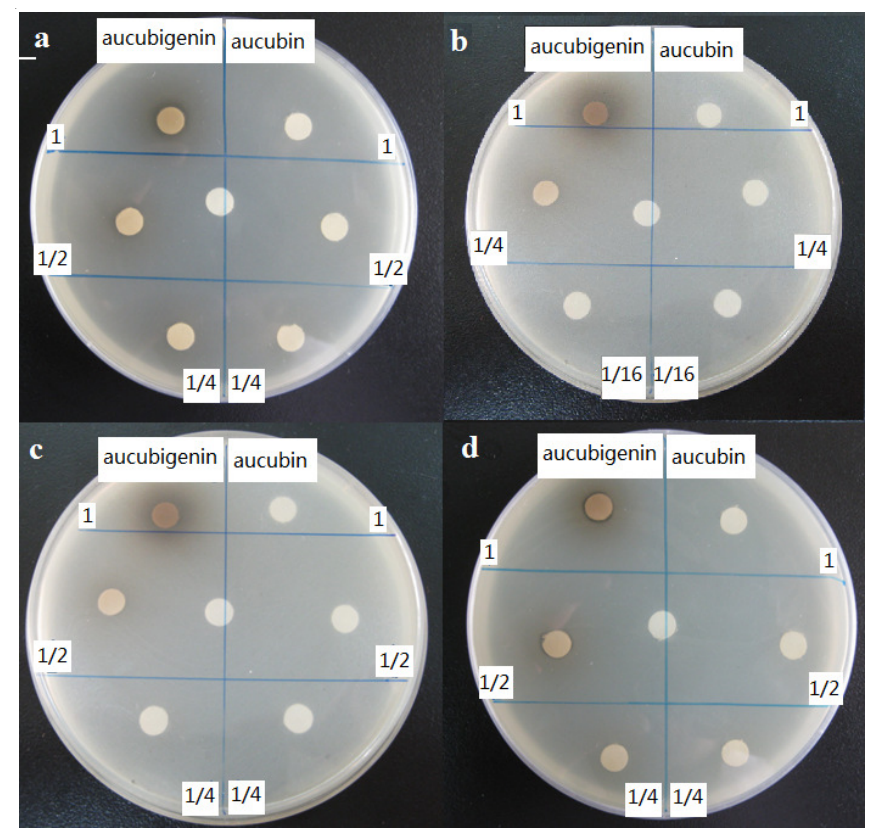

Fig. 1. Inhibitory effect of aucubigenin and aucubin by the disc diffusion method. a: Escherichia coli (ATCC 25922); b: Staphylococcus aureus (ATCC 25923); c: Salmonella enterica (ATCC 14028); d: Pseudomonas aeruginosa (ATCC 27853)

results indicated that aucubigenin exhibited considerable antibacterial activity against all tested strains and was particularly more effective against Staphylococcus aureus, the MIC value was $0.0313 \mathrm{mg} / \mathrm{mL}$ and the MBC value was $0.0625 \mathrm{mg}$ / $\mathrm{mL}$ (Fig. 2c). Aucubigenin showed moderate antibacterial activity against Escherichia coli and Salmonella typhi with MIC of $1 \mathrm{mg} / \mathrm{mL}$ and MBC of $2 \mathrm{mg} / \mathrm{mL}$, respectively (Fig. 2a and 2d). Aucubigenin was less effective against Pseudomonas aeruginosa with both MIC and MBC of $2 \mathrm{mg} / \mathrm{mL}$ (Fig. 2b). As expected, aucubin had no inhibitory activity against the upgrowth of all tested strains.

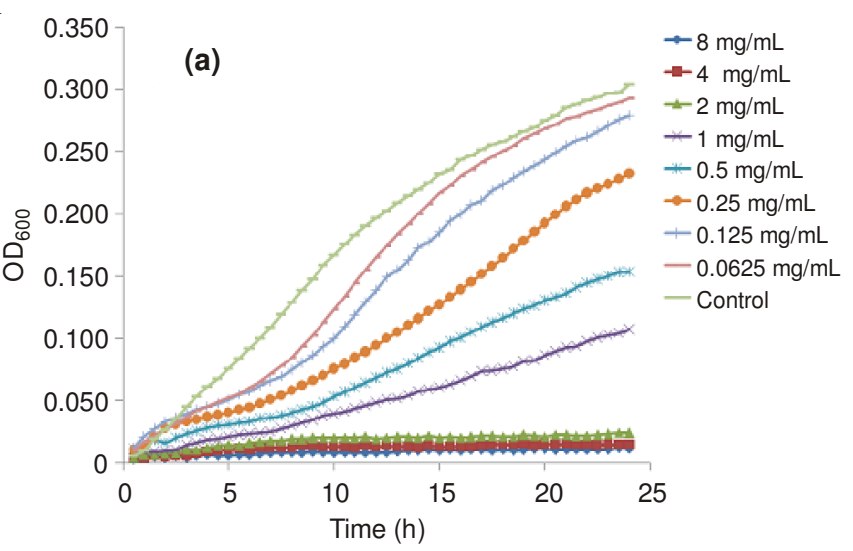

TABLE-1

ANTIBACTERIAL ACTIVITY OF AUCUBIGENIN AND AUCUBIN AGAINST THE BACTERIAL STRAINS

\begin{tabular}{ccccc}
\hline Microorganisms & $\mathrm{MIC}(\mathrm{mg} / \mathrm{mL})$ & $\mathrm{MBC}(\mathrm{mg} / \mathrm{mL})$ & $\mathrm{MIC}(\mathrm{mg} / \mathrm{mL})$ & $\mathrm{MBC}(\mathrm{mg} / \mathrm{mL})$ \\
\hline Escherichia coli & 1 & 2 & - & - \\
Staphylococcus aureus & 0.0313 & 0.0625 & - & - \\
Pseudomonas aeruginosa & 2 & 2 & - & - \\
Salmonella enterica & 1 & 2 & - & - \\
\hline
\end{tabular}

* A dash (-) indicate no antibacterial activity 

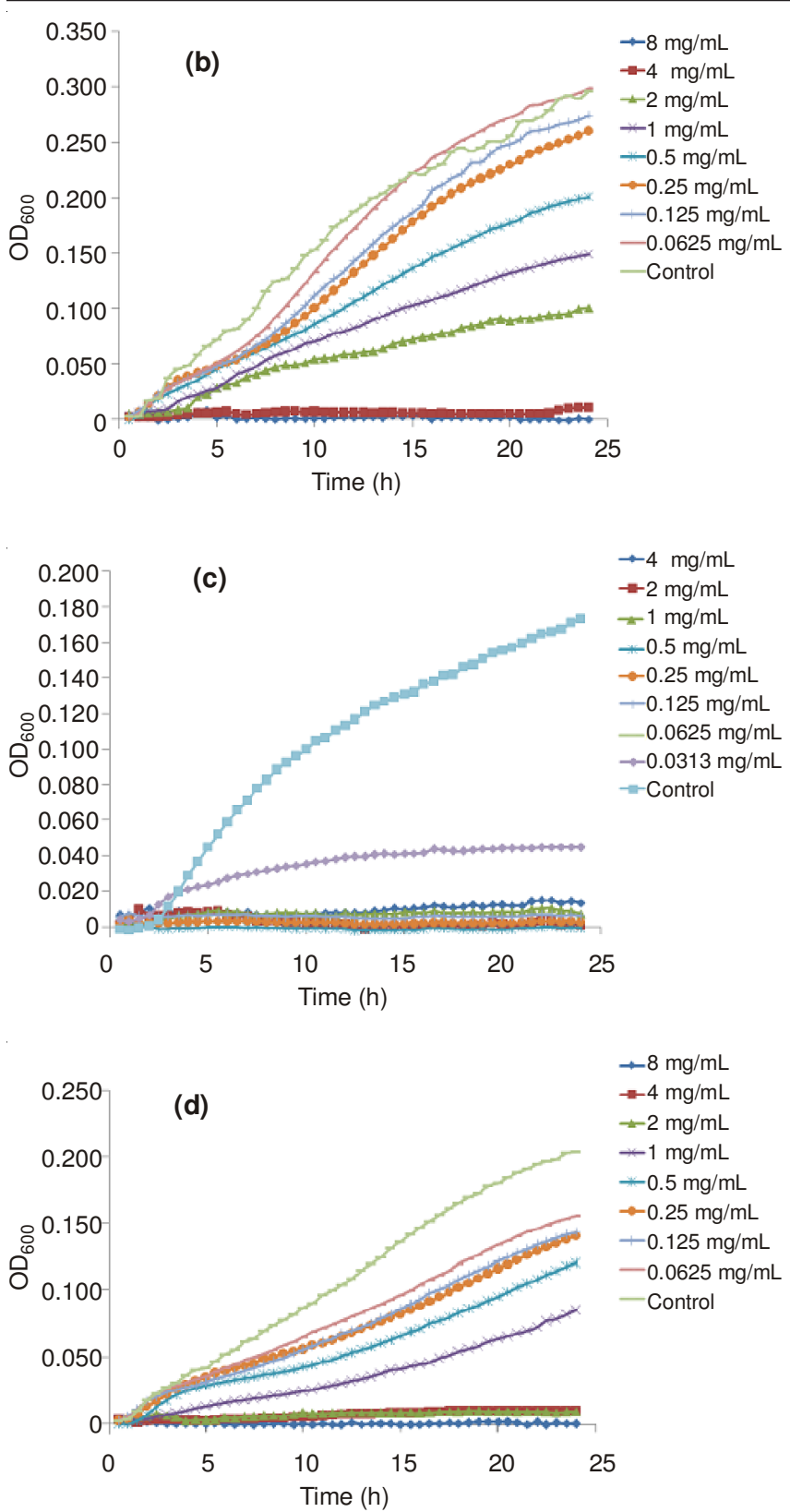

Fig. 2. Micro-well dilution assay of aucubigenin a: Escherichia coli (ATCC 25922); b: Pseudomonas aeruginosa (ATCC 27853); c: Staphylococcus aureus (ATCC 25923); d: Salmonella enterica (ATCC 14028).

\section{Conclusion}

In this study, a sensitive standardized micro-well dilution with automated spectrophotometric method was used to determine the antibacterial activity of natural iridoid glucoside aucubin and its aglycone. The results demonstrated that the micro-well dilution assay was more accurate than paper disc diffusion method. Aucubigenin exhibited appreciable antibacterial activity and is particularly more effective against Staphylococcus aureus, while aucubin did not show antibacterial activity.

\section{ACKNOWLEDGEMENTS}

This study was financially supported by National Natural Science Foundation of China (No. 31200253), Natural Science Basic Research Plan in Shaanxi Province of China (No. 2012JQ4031), Shaanxi Provincial Education Department (No. 2013JK0810) and Specialized Research Fund for the Doctoral Program of Higher Education (No. 20126101120016).

\section{REFERENCES}

1. H.Y. Kil, E.S. Seong, B.K. Ghimire, I.M. Chung, S.S. Kwon, E.J. Goh, K. Heo, M.J. Kim, J.D. Lim, D. Lee and C.Y. Yu, Food Chem., 115, 1234 (2009).

2. J. Santas, M. Almajano and R. Carbo, Int. J. Food Sci. Technol., 45, 403 (2010)

3. Y. Li, Y. Zhao, Y.M. Zhang, M.J. Wang and W.J. Sun, Carbohydr. Res., 344, 2270 (2009).

4. A. Bianco, M. Guiso, C. Iavarone, P. Passacantilli and C. Trogolo, Tetrahedron, 33, 847 (1977).

5. K. Ishiguro, M. Yamaki and S. Takagi, Yakuguku Zassi, 102, 755 (1982).

6. E. Davini, C. Javarone, C. Trogolo, P. Aureli and B. Pasolini, Phytochemistry, 25, 2420 (1986).

7. C. Llop, I. Pujol, C. Aguilar, J. Sala, D. Riba and J. Guarro, Antimicrob. Agents Ch., 44, 239 (2000).

8. M.H. Oliver, N.K. Harrison, J.E. Bishop, P.J. Cole and G.J. Laurent, J. Cell Sci., 92, 513 (1989).

9. A. Bamoniri, A.H. Ebrahimabadi, A. Mazoochi, M. Behpour, F.J. Kashi and H. Batooli, Food Chem., 122, 553 (2010).

10. M. Gulluce, A. Aslan, M. Sokmen, F. Sahin, A. Adiguzel, G. Agar and A. Sokmen, Phytomedicine, 13, 515 (2006).

11. P.S. Pavithra, N. Sreevidya and R.S. Verma, J. Ethnopharmacol., 124, 151 (2009). 